\title{
Azimuthal integration and crystallographic algorithms on field-programmable gate arrays
}

\author{
Z. Matěj ${ }^{1}$, K. Skovhede ${ }^{1,2}$, C. Johnsen ${ }^{2}$, A. Barczyk ${ }^{1}$, C. Weninger ${ }^{1}$, A. Salnikov ${ }^{1}$, B. Vinter ${ }^{2}$ \\ ${ }^{1}$ MAX IV Laboratory, Lund University, Sweden, \\ ${ }^{2}$ Niels Bohr Institute, University of Copenhagen, Denmark
}

zdenek.matej@maxiv.lu.se

With the onset of next generation synchrotron light sources and X-ray free electron lasers, accompanied by developments of future photon detectors, delivering mega-pixel diffraction images at frame rates over $10 \mathrm{kHz}$, production of data from crystallography experiments is rapidly increasing. Crystallographers were utilizing various types of computing hardware from the early beginning. Algorithms and computing devices were constantly developing. Nowadays even quantum computers are available in commercial clouds. A potential of Field-Programmable Gate Arrays (FPGAs) - a more classical computing accelerators, is explored and demonstrated in this work on a task of Azimuthal Integration (AZINT) of area-detector data from powder diffraction and small angle scattering. Beside these two most known application cases, where among other data volumes are reduced by a factor of 1000 , the underlaying procedure i.e. bin-counting has applications in data analysis tasks as background estimation in conventional single crystal diffraction images or data reduction from diffraction anomalous fine structure. The new potential of FPGAs for big data science and complex data analysis originates from recent progress in tools allowing scientific software developers to easily program FPGAs, prototype and implement algorithms on them with complexity fitting the scientific requirements. It is demonstrated that AZINT can process $600 \mathrm{~Gb} / \mathrm{s}$ of uncompressed data stream, i.e. about 20-40 Gpixels/s, on a single commercial FPGA available at photon and neutron facilities or compute clouds, however energy and cost-effective commodity hardware FPGAs can be used as well. FPGAs are usually more energy-efficient in comparison to widely known graphical processing units (GPUs) and they are very flexible so they can better fit a specific problem and outperform GPUs in many relevant applications, in particular AZINT here. Beside high throughput required for big data analysis FPGAs allow data reduction and filtering with well-defined and low latencies. This enables experiments with X-rays as a real-time probe. Development of crystallographic code for big data handling on FPGAs may have additional synergies. FPGAs can be radiation tolerant and operate under some extreme conditions. It makes them ideal components for extraterrestrial crystallography (e.g. Mars rovers). AZINT was developed at MAX IV synchrotron Laboratory however similar activities are present on other photon sources. It is worth to mention at least data acquisition and spot-finding project [1] for macromolecular crystallography at SLS/PSI.

[1] Leonarski, F., Mozzanica, A., Brückner, M., Lopez-Cuenca, C., Redford, S., Sala, L., Babic, A., Billich, H., Bunk, O., Schmitt, B., Wang, M. (2020). Structural Dynamics 7, 014305. doi: $10.1063 / 1.5143480$

Keywords: computing, diffraction, azimuthal integration, big data, light sources

MAX IV Laboratory and its funders are kindly acknowledged. Research conducted at MAX IV, a Swedish national user facility, is supported by the Swedish Research council under contract 2018-07152, the Swedish Governmental Agency for Innovation Systems under contract 2018-04969, and Formas under contract 2019-02496". 